Trauma Berufskrankh 2016 · [Suppl 2]: 18:S203-S207

DOI 10.1007/s10039-015-0113-6

Online publiziert: 26. Januar 2016

๑) Springer-Verlag Berlin Heidelberg 2016

CrossMark

\section{Pseudarthrose („,non union“)}

Stellt sich bei der Knochenheilung keine knöcherne Konsolidierung ein („non union“) wird dies ab einem Zeitraum von 6 Monaten als Pseudarthrose bezeichnet. Diese Komplikation der Frakturheilung betrifft zwischen 3 und $10 \%$ aller Patienten. Hochgerechnete Daten aus dem schottischen National Health Service (NHS) lassen eine Häufigkeit von ca. 200.000 neu aufgetretenen Pseudarthrosen pro Jahr in Europa annehmen [1]. Dabei werden hypertrophe Pseudarthrosen mit überschießender Knochenneubildung, meist verursacht durch unzureichende Retention der Frakturenden, von hypotrophen und atrophen Pseudarthrosen unterschieden [2]. Bei der Therapie der hypertrophen Pseudarthrosen reicht meist die suf-

\author{
G. Täger ${ }^{1} \cdot$ F. Hilger $^{1} \cdot$ U. Seifritz ${ }^{1}$ L. Podleska ${ }^{2}$ \\ ${ }^{1}$ Klinik für Orthopädie und Unfallchirurgie, Hand- und Wiederherstellungschirurgie, \\ Klinikum Kempten, Kempten, Deutschland \\ ${ }^{2}$ Zentrum für Chirurgie, Universitätsklinikum Essen, Essen, Deutschland
}

\title{
Knochenaugmentation bei hypo- und atrophen Pseudarthrosen
}

fiziente Reosteosynthese aus (z. B. Austauschmarknagelung, Augmentations-, Kompressionsplatte), um die vorhandene Knochenneubildung zur knöchernen Konsolidierung der Frakturen nutzen zu können [3].

Dagegen stehen bei hypo- und atrophen Pseudarthrosen neben der Notwendigkeit einer Reosteosynthese besonders die Konduktion (Matrixstruktur) und die Induktion (Neubildung) der Knochenstruktur im Mittelpunkt. Ebenso ist aber der Ausschluss einer Infektion unbedingt erforderlich, um niedriggradige, klinisch inapparente Infektionen detektieren zu können. Dazu ist die Gewebebiopsie aus der Pseudarthrose erforderlich (• Abb. 1). Die Durchführung molekularbiologischer Untersuchungen [Polymerasekettenreaktion (PCR)] ist dabei sinnvoll, um über den Nachweis von Bruchstücken der Erbsubstanz die jeweiligen Bakterien identifizieren und die antimikrobielle Therapiestrategie festlegen zu können [4].

Unter dem Begriff Augmentation werden zur Erstellung von Therapiekonzepten bei hypo- und atrophen Pseudarthrosen alle Maßnahmen subsumiert, die entsprechend der Etymologie („augmentare“: vermehren, fördern) zur Knochenneubildung und Knochenheilung beitragen. An diese Strategie lehnt sich auch das von Giannoudis et al. [5] publizierte „diamond concept“ an. Allerdings sind in diesem chirurgischen Konzept die adjuvant einsetzbaren physikalischen Möglichkeiten (extrakorporale Stoßwelle, niedrig gepulster Ultraschall) nicht berücksichtigt.

Nachfolgend werden die grundsätzlich zur Verfügung stehenden Möglichkeiten
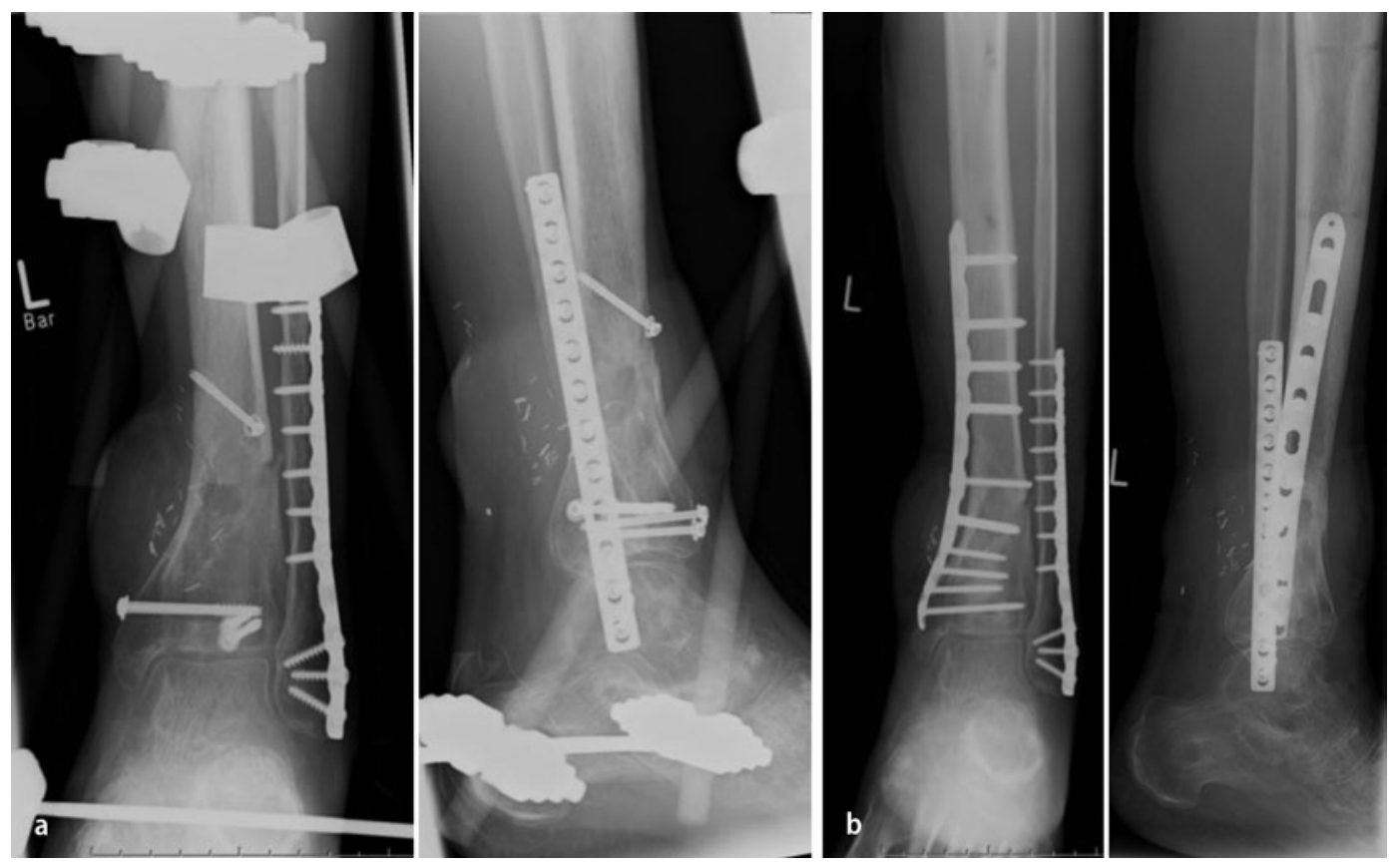

Abb. $1<$ Hypotrophe Pseudarthrose der distalen Tibia (47 Jahre, männlich). a Verfahrenswechsel nach distaler Tibiafraktur $\mathrm{G} \|^{\circ}$ mit primärer Plattenosteosynthese, Frühinfekt und Hautnekrose, mehrzeitigen Operationen (Metallentfernung, Débridement, Fixateur externe, freie Anterior-lateral-thighLappenplastik). b Dekortikation, autologe Spongiosaplastik, Plattenosteosynthese mit Ausheilung 

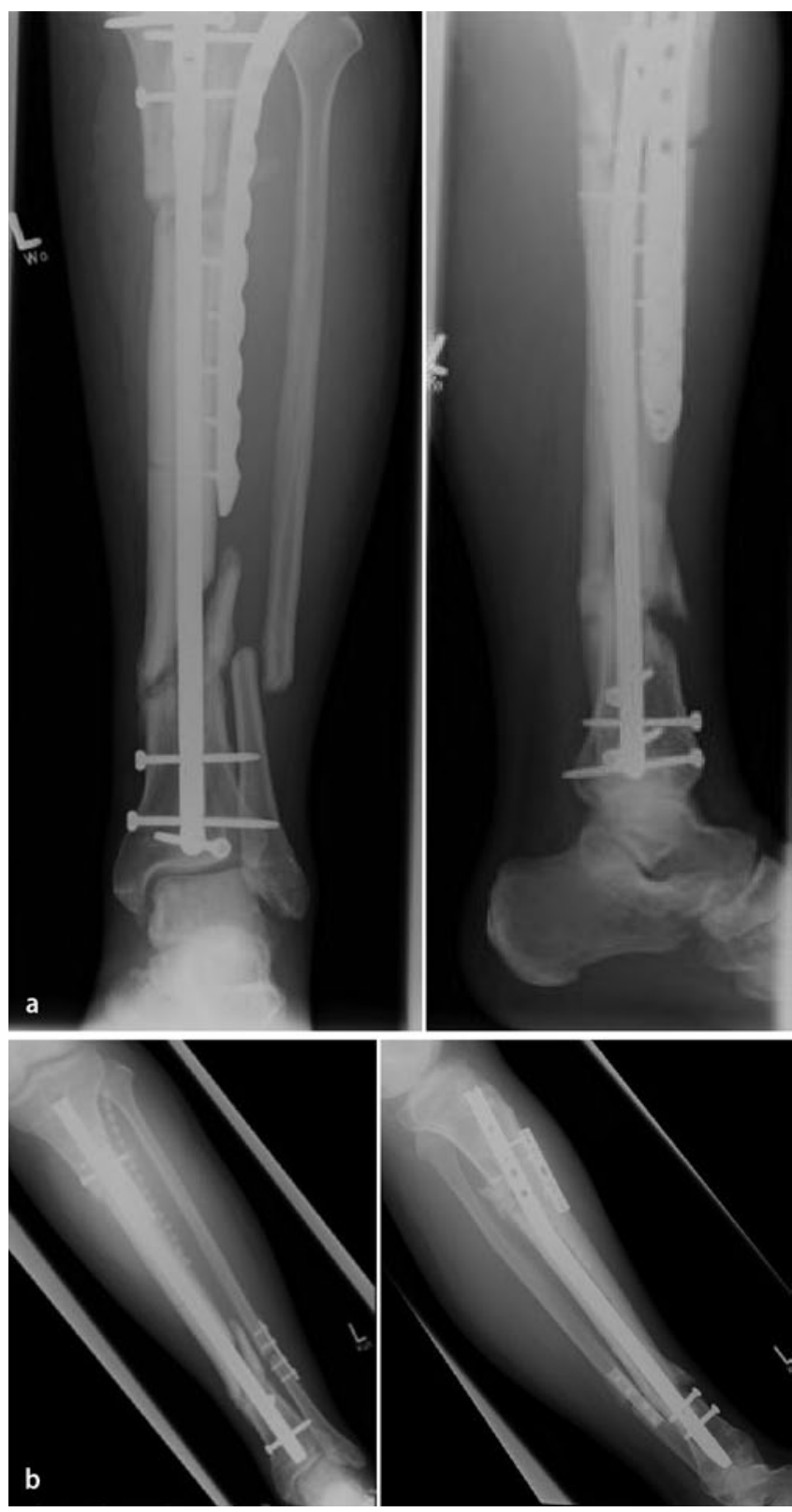

Abb. $2<$ a Hypotrophe Pseudarthrose der distalen Tibia (47 Jahre, männlich) nach Etagenfraktur der Tibia (proximal III distal ${ }^{\circ}$ geschlossen) mit kombinierter Osteosynthese (Platte und Marknagel). b Verfahrenswechsel mit Marknagelentfernung, gebohrter Marknagelung mit innerer Spongiosaplastik, Fibulaosteotomie

im Hinblick auf ihre Anwendbarkeit und Kombinationsfähigkeit sowie auch im Hinblick auf ihre Ergebnisse dargestellt.

\section{Nichtoperative Therapiemöglichkeiten}

\section{Niedrig gepulster Ultraschall und extrakorporale Stoßwellentherapie}

Beide Verfahren basieren auf dem Prinzip der physikalischen Beeinflussung stromabildender Zellen. Die physikalischen Grundlagen der beiden Anwendungen LIPUS („low intensity pulsed ultrasound“) und ESWT („extracorporal bildung [6].
Sowohl die für EWST wie auch die für LIPUS publizierten klinischen Daten berichten über erfolgreiche Knochenheilungen zwischen 50 und $80 \%$ ohne weitere chirurgische Maßnahmen. Allerdings handelt es sich dabei um retrospektive Kohortenstudien, die eine sehr hohe Varianz für Indikationen, patientenbezogene Faktoren und Durchführung aufweisen [6]. Schoellner et al. [7] haben in ihrer Untersuchung von 43 Pseudarthrosen herausgearbeitet, dass die Heilungswahrscheinlichkeit nach ESWT umso höher ist, umso mehr Stoffwechselaktivität szintigraphisch noch nachweisbar ist. Daher scheint es sinnvoll zu sein, diese Behandlungsmodalität auch zu einem frühen Zeitpunkt einzusetzen, um in dem noch proliferierenden Gewebe auch die Heilungseffekte der physikalisch nachgewiesenen Mechanismen erreichen zu können. Anders herum betrachtet, scheinen die Patienten mit szintigraphisch negativen Befunden nicht von der Durchführung der ESWT/LIPUS zu profitieren [7].

Obwohl der positive Effekt der EWST in experimentellen Untersuchungen klar nachgewiesen werden konnte [6], fehlt aber der wissenschaftliche Beweis für die klinische Effektivität der EWST und auch des LIPUS bis heute. Deswegen sind diese Behandlungsmöglichkeiten nach wie vor nicht Bestandteil des GKV (gesetzliche Krankenversicherung)-Katalogs und auch nicht Gegenstand einer neuerlichen Bewertung durch den GBA (Gemeinsamer Bundesausschuss).

\section{Operative \\ Therapiemöglichkeiten}

sich zwar dennoch ist beiden die Aktivierung der Wundheilungssituation mittels mechanischer Stimulation durch Applikation lokaler Zug- und Dehnungskräfte gemein. Entscheidend sind dabei einerseits die Hyperpolarisierung der Zellmembranen und andererseits die durch die Druck- und Kavitation entstehenden Mikrofrakturierungen des Knochengewebes wie auch Mikroschädigungen des Stromagewebes. Dadurch erfolgt die Induktion der Wundheilungsvorgänge der frühen Phasen (Proliferation, Neoangiogenese) mit konsekutiver Knochen-

\section{Autologes Knochentransplantat}

Die Transplantation von autologem Knochen gilt nach wie vor als Therapiestandard bei der Behandlung hypound atropher Pseudarthrosen. Sie führt in über $95 \%$ der Anwendungen zur erfolgreichen Knochenheilung [8] und kann als sog. innere Spongiosaplastik bei simultaner Markraumbohrung (• Abb. 2) oder als freie Transplantation von autologer Spongiosa vorsam ist, dass mit dem operativen Eingenommen werden (• Abb. 3). Bedeut- 
griff bei autologer Spongiosaplastik als solchem die Anfrischung sowohl des periossalen Gewebes (Induktion der Wundheilung) wie auch des knöchernen Gewebes (Dekortikation, Induktion der Wund-, Knochenheilung) verbunden ist (- Abb. 2). In der Hauptsache beinhaltet die autologe Knochentransplantation aber die Voraussetzungen, die für das Erreichen einer knöchernen Heilung gegeben sein müssen und wie sie von Giannoudis et al. [5] publiziert worden sind: die Osteokonduktion in Form der Matrixstruktur der Knochenpartikel und die Osteoinduktion in Form der im autologen Transplantat enthaltenen osteogenen Zellen und Wachstumsfaktoren [8].

Im Hinblick darauf, welche Menge an autologem Knochen mit welchem Zeitaufwand gewonnen werden kann, haben Dawson et al. [9] die Transplantatentnahme am vorderen Beckenkamm, am hinteren Beckenkamm und mittels RIA („reaming irrigation/aspiration“)Technik vergleichend untersucht. Dabei haben sie festgestellt dass am vorderen Beckenkamm durchschnittlich $20 \mathrm{~cm}^{3}$ gewonnen werden können gegenüber $36 \mathrm{~cm}^{3}$ am hinteren Beckenkamm und $38 \mathrm{~cm}^{3}$ bei Anwendung der endofemoralen Bohrtechnik. Der Zeitaufwand und das Outcome im Hinblick auf die Morbidität waren ähnlich [9].

\section{„Bone morphogenic proteins"}

Die einzig zugelassene Indikation bei der Pseudarthrosenbehandlung an den metaund diaphysären Skelettabschnitten ist für Frakturen der proximalen Tibia definiert. Alle anderen Anwendungen stellen Einzelfallindikationen dar und sind nicht Gegenstand des GKV-Katalogs. Entsprechend zurückhaltend ist die Einschätzung durch den Medizinischen Dienst der Krankenkassen im Hinblick auf die Kostenerstattung. Tatsächlich ist unter Berücksichtigung der publizierten Untersuchungen zur Frakturheilung weder in der Primärsituation noch in der Pseudarthrose ein Vorteil durch Anwendung von „bone morphogenic proteins“ (BMP) erkennbar $[10,11]$. Dies ist nachvollziehbar, weil die alleinige lokale Applikation von BMP auf ausdifferenziertem und

Trauma Berufskrankh 2016 · [Suppl 2]: 18:S203-S207 DOI 10.1007/s10039-015-0113-6

๑) Springer-Verlag Berlin Heidelberg 2016

\section{G. Täger · F. Hilger · U. Seifritz · L. Podleska}

\section{Knochenaugmentation bei hypo- und atrophen Pseudarthrosen}

\section{Zusammenfassung}

Das Auftreten einer verzögerten Frakturheilung wird im deutschsprachigen Raum traditionell nach einem Zeitraum von 6 Monaten mit dem Begriff der Pseudarthrose belegt. Das Risiko für diese schwerwiegende Komplikation wird mit Häufigkeiten zwischen 3 und $10 \%$ angegeben. Das Auftreten wird unter anderem durch die Determinanten Frakturmorphologie, Weichgewebeschaden, Osteosynthesetechnik sowie durch patientenbezogene Faktoren (Lokalisation, Alter, Komorbidität, Medikamente, Nikotin) definiert. Zur Behandlung werden ganz grundsätzlich diejenigen

\begin{abstract}
Pseudarthrosen mit ausreichender/überschießender Knochenneubildung (hypertrophe Pseudarthrose) von denen mit ungenügender oder ganz ausbleibender Knochenheilung (hypotrophe Pseudarthrose) unterschieden. Für die hypo- und atrophen Pseudarthrosen werden in dieser Übersicht die Möglichkeiten zur Augmentation des geschädigten und nicht heilen wollenden Knochens aufgezeigt.
\end{abstract}

Schlüsselwörter

Knochen · Fraktur · Komplikation ·

Osteosynthese $\cdot$ Augmentation

\section{Bone augmentation in hypotrophic and atrophic pseudarthroses}

\section{Abstract}

In German-speaking countries the occurrence of delayed healing of fractures after a period of 6 months is traditionally known by the term pseudarthrosis. The risk for this severe complication is reported as having a frequency between $3 \%$ and $10 \%$. The occurrence is predominantly defined by the determinants fracture morphology, soft tissue damage and osteosynthesis technique as well as by patient-related factors, such as localization, age, comorbidities, medication and nicotine consumption. For treatment a fundamental differentiation is made between pseudarthro- sis with sufficient or superfluous bone regeneration (hypertrophic pseudarthrosis) and pseudarthrosis with insufficient (hypotrophic pseudarthrosis) or complete lack of bone healing (atrophic pseudarthrosis). This review article presents the options for augmentation of damaged bone and bone that does not heal in hypotrophic and atrophic pseudarthroses.

Keywords

Bones · Fracture - Complication . Osteosynthesis · Augmentation bradytrophem Narbengewebe nicht zur weiteren Ausdifferenzierung von knochenbildenden Zellen führen kann. Zuletzt haben Takemoto et al. [11] in einer konsekutiven Untersuchung die Bedeutung von rhBMP-2 in der Therapie von 118 Pseudarthrosen untersucht. Dabei haben sie im Vergleich bei Anwendung von autologer Spongiosa gegenüber der Verwendung von rhBMP-2 („recombinant human bone morphogenic protein 2“) mit Spongiosa keinen Vorteil für die mit rhBMP-2 behandelten Patienten erkennen können. Damit bleibt die Anwendung von rhBMP tatsächlich den Situationen vorbehalten, in denen mit den oben genannten und etablierten Maßnahmen nicht ausreichend sicher die knöcherne Heilung erreicht werden kann (Individualentscheidung).

\section{Knochenersatzstoffe}

(allogene Transplantate, „platelet rich plasma“)

Allogene Spongiosatransplantate haben bei der Behandlung hypo- und atropher diaphysärer Pseudarthrosen keinen erkennbaren Stellenwert, obwohl sie in beliebiger Menge und ohne Entnahmemorbidität verfügbar wären. Der mangelnde Stellenwert zeigt sich daran, dass in der Literatur der letzten Jahre zu allogenen Transplantaten bei diaphysären Pseudarthrosen keine Untersuchungen publiziert worden sind. Grund dafür ist, dass allogene Transplantate ausschließlich als Matrixstruktur fungieren und die Mechanismen der Osteoinduktion fehlen.

Im Gegensatz dazu wird in aktuellen Publikationen die Anwendung von 

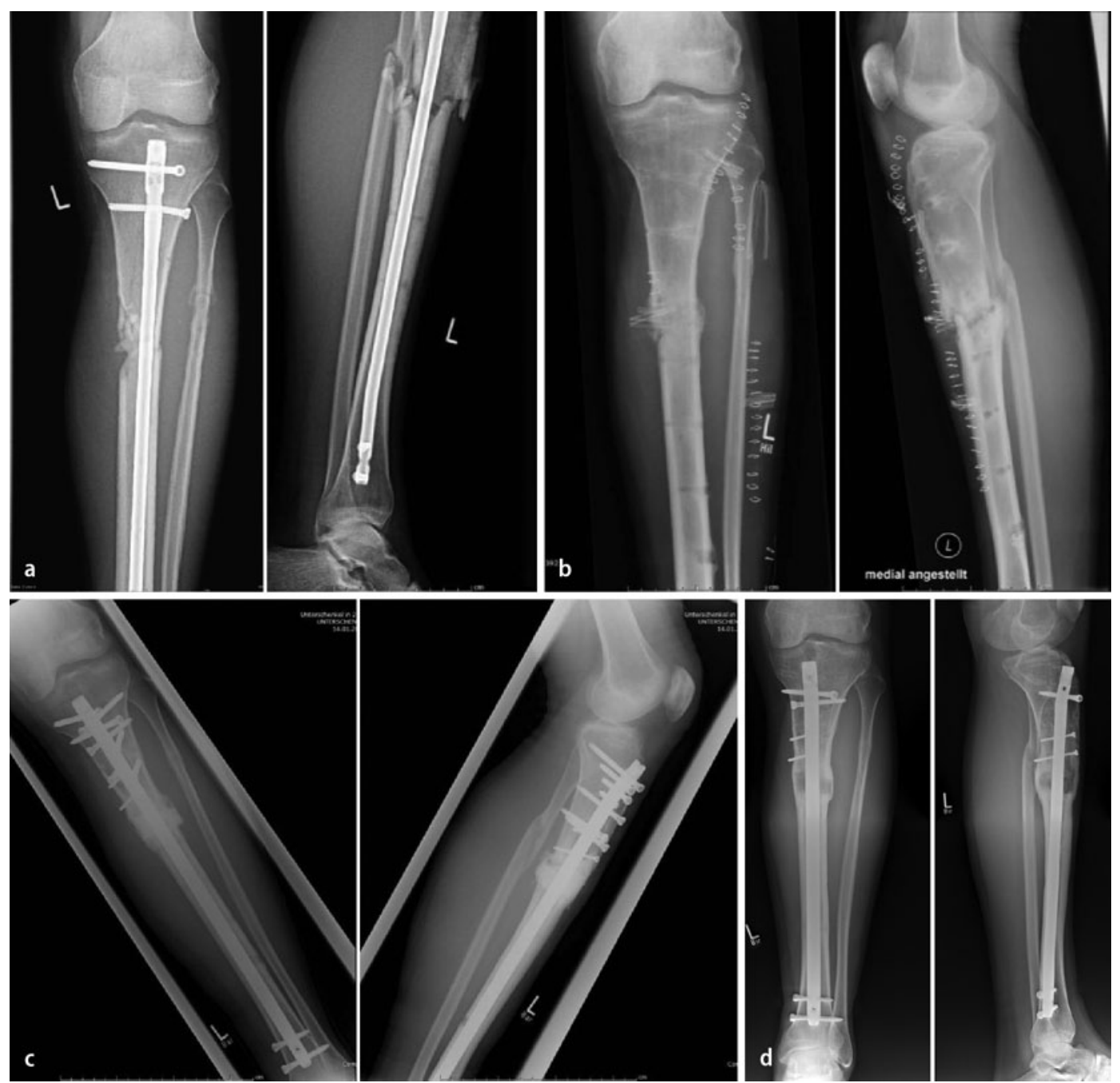

Abb. $3<$ Defektpseudarthrose der proximalen Tibia mit mehrzeitigem Behandlungsvorgehen und Knochenersatzverfahren nach Masquelet (24 Jahre, männlich). a Pseudarthrose nach Marknagelosteosynthese. b Pseudarthrose nach erfolglosem Verfahrenswechsel und Entfernung der winkelstabilen Platte sowie Biopsie (Ausschluss Infektion). c Verfahrenswechsel mit Marknagelosteosynthese, Pollerschrauben und Platzhalter [Polymethylmetacrylat (PMMA)]. d Knöcherne Konsolidierung nach Entfernung des PMMA-Platzhalters und autologer Knochentransplantation sowie Injektion von "platelet rich plasma“ thrombozytenreichem Plasma („platelet rich plasma“, PRP) als weitere Facette der Behandlungsmöglichkeiten dargestellt $[12,13]$. Abgesehen von der Tatsache, dass thrombozytenreiches Plasma bereits für eine Vielzahl von Indikationen in der muskuloskeletalen Medizin angewendet wird, sind in den genannten Arbeiten positive Effekte auf die Knochenheilung bei Knochendefekten und Pseudarthrosen beschrieben. Neben der von Hakimi et al. [13] bei 17 Patienten beobachteten Ausheilung der Pseudarthrose durch Anwendung von PRP mit autologer Spongiosa sind die nachgewiesenen hohen Konzentrationen von Thrombozytenzahl, PDGF- und TGF- $\beta 1$-Konzentration bemerkenswert. Obwohl auch Galasso et al. [14] in einer Untersuchung an 22 Patienten bei diaphysärer Pseudarthrose durch aufgebohrte Marknagelung in Verbindung mit PRP-Applikation die Ausheilung bei 20 Patienten berichten, stellt auch diese Anwendungsform bisher nur eine mögliche zusätzliche Option neben der Verwendung autologer Spongiosa dar.

Diese zusätzliche Option ist aufgrund ihrer geringen Invasivität und aufgrund der nachgewiesenermaßen hohen Konzentration an Thrombozyten, vitalen pluripotenten Stammzellen und Wachstumsfaktoren (PDGF, TGF- $\beta 1$ ) sehr vielversprechend. In der eigenen Anwendung ist dieses Verfahren etabliert und wird als zusätzliche Maßnahme der Anwendung von rhBMP-2 vorgezogen (- Abb. 1).

Die Vitalisierung von allogenem spongiösem Knochen durch Zusatz von PRP kann eine Weiterentwicklung der bisher etablierten Behandlungskonzepte bedeuten.

\section{Schlussfolgerung}

Hypo- und atrophe Pseudarthrosen sind durch das Vorliegen von fibrozytärem Stroma mit hohem mesenchymalem Differenzierungsgrad, geringer Gefäßdichte und Verlust neuronaler Proliferation gekennzeichnet. Dies stellt den Endzustand der Gewebedifferenzierung im Rahmen der Heilungsvorgänge dar, die eigentlich zu Gewebeproliferation und knöcherner Heilung führen sollten. Das Ziel der Behandlung dieser Pseudarthrosen liegt deswegen grundsätzlich darin, einen neuen Impuls zu geben, der die Gewebeproliferation und spätere Differenzierung anstößt. Infektionen müssen ausgeschlossen sein, weshalb die Biopsie der Pseudarthrose empfohlen wird.

Mit den physikalischen Therapiemöglichkeiten können nur bei etwas über 
der Hälfte der Pseudarthrosen knöcherne Konsolidierungen erreicht werden. Die frühe Anwendung scheint sinnvoll zu sein. Bei den operativen Verfahren bleibt die Dekortikation mit autologer Spongiosatransplantation das Maß der Dinge. Die Anwendung von rhBMP-2 ist mit großer Zurückhaltung zu bewerten, die Applikation von thrombozytenreichem Plasma ist eine interessante Option, aber bislang unzureichend untersucht.

\section{Korrespondenzadresse}

\section{Prof. Dr. G. Täger}

Klinik für Orthopädie und Unfallchirurgie, Handund Wiederherstellungschirurgie,

Klinikum Kempten

Robert-Weixler-Str. 50, 87439 Kempten

Georg.Taeger@klinikum-kempten.de

\section{Einhaltung ethischer Richtlinien}

Interessenkonflikt. G. Täger ist als Referent und Berater bei den Firmen Smith\&Nephew sowie Zimmer tätig. F. Hilger und L. Podleska geben an, dass kein Interessenkonflikt besteht.

Dieser Beitrag beinhaltet keine Studien an Menschen oder Tieren.

Alle Patienten, die über Bildmaterial oder anderweitige Angaben innerhalb des Manuskripts zu identifizieren sind, haben hierzu ihre schriftliche Einwilligung gegeben.

The supplement containing this article is not sponsored by industry.

\section{Literatur}

1. ICD-10 coding, source SMR01. Glasgow, Health Services Information Group, ISD, NHS National Services, Scotland: 2011

2. Weber B, Czech O (1973) Pseudoarthrosen: Pathophysiologie, Biomechanik, Therapie, Ergebnisse, 1. Aufl. Huber, Bern

3. Tsang ST, Mills LA, Baren J, Frantzias J, Keating JF, Simpson AHRW (2015) Exchange nailing for femoral diaphyseal fracture non union: Risk factors for failure. Injury. http://dx.doi.org/10/1016/j. injury.2015.09.027

4. Gille J, Wallstabe S, Schulz AP, Paech A, Gerlach U (2012) Is non union of tibial sgaft fractures due to nonculuturable bacterial pathogens? A clinical investigation using PCR and cultur techniques. J Orthop Res 7(20):1749-1799

5. Giannoudis P, Einhorn TA, Marsh D (2007) Fracture healing: the diamond concept. Injury 3854:53-56

6. Maier M, Milz S, Tischer T (2002) Influence of extracorporal shock-wave-application on normal bone in an animal model in vivo: scintography, $\mathrm{MRI}$ and histopathology. J Bone Joint Surg $\mathrm{Br}$ 64:592-599
7. Schoellner C, Rompe JD, Decking J, Heine J (2002) Die hochenergetisch extrakorporale Stoßwellentherapie (ESWT) bei Pseudarthrose. Orthopäde 31:658-662

8. Sen MK, Miclau T (2007) Autologous iliac crest bone graft: should it still be the gold standard for treating non-unions? Injury 38(Suppl 1):S75-S80

9. Dawson J, Kiner D, Gardner W 2nd, Swafford R, Nowotarski PJ (2014) The reamer-irrigatoraspirator as a device for harvesting bone-graft compared with iliac crest bone graft: union rates and complications. J Orthop Trauma 10(28):584590

10. Garrison KR, Shemilt I, Donell S, Ryder JJ, Mugford M, Harvey I, Song F, Alt V (2010) Bone Morphogenic Protein (BMP) for fracture healing in adults. Cochrane Database Syst Review. doi:10.1002/14651858

11. Takemoto R, Forman J, Taormina DP, Egol KA (2014) No advantage to rhBMP-2 in addition to autogenous bone graft for fracture non-union. Orthopedics 37(6):e525-e530

12. Marx RE, Carlson ER, Eichstaedt RM (1998) Plateletrich-plasma growth factor enhancement for bone grafts. Oral Surg Oral Medical Oral Pathol Oral Radiol Endod 85:638-646

13. Hakimi $M$, Jungbluth $P$, Thelen $S$, Betsch $M$, Linhart W, Flohé S, Windolf J, Wild M (2011) Platelet-richplasma in Kombination mit autologer Spongiosa. Unfallchirurg 114(11):998-1006

14. Galasso O, Mariconda M, Romano G, Capuano N, Romano L, lannò B, Milano C (2008) Expandable intramedullary nailing and platelet rich plasma to treat long bone non-unions. J Orthop Traumatol 9(3):129-134 
